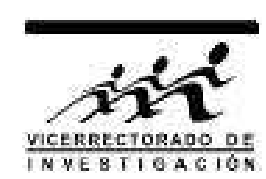

\title{
Modelo físico de la estimación de la concentración de clorofila-a en el mar peruano usando imágenes MODIS
}

\author{
M. G. Quezada García ${ }^{1}$, R. I. Paredes Peñafiel ${ }^{1}$, F. Carrillo Gomero² y J. Rojas Acuña ${ }^{3 *}$ \\ ${ }^{1}$ Facultad de Ciencias Biológicas, ${ }^{2}$ Facultad de Ingeniería Electrónica y ${ }^{3}$ Facultad de Ciencias Físicas, \\ Universidad Nacional Mayor de San Marcos, Av. Venezuela s/n, Lima 1, Perú
}

Recibido 15 enero 2011 - Aceptado 28 diciembre 2011

\begin{abstract}
La clorofila-a es un indicador de la biomasa del plancton para rastrear corrientes, chorros y plumas oceanográficas, y se utiliza como un parámetro de entrada en los modelos de producción primaria. La clorofila a de la superficie del mar peruano puede ser medida mediante la teledetección por satélite en el rango espectral del visible ubicado entre 0.4 y $0.7 \mu \mathrm{m}$. El objetivo de presente trabajo es desarrollar un modelo físico para la estimación de la concentración de la clorofila-a procedente de las imágenes MODIS en el mar peruano

Palabras claves: Clorofila, imágenes por satélite, MODIS, oceanografía.
\end{abstract}

\section{A physical model of the estimation of the chlorophill a in the Peruvian sea using MODIS images}

The chlorophyll a is an indicator of the plankton biomass for track flows, jets and oceanographic feathers and is used as an entry for primary production models. The chlorophyll a in the Peruvian sea surface can be measured by means of satellite remote sensing in the visible spectral range from 0.4 to $0.7 \mu \mathrm{m}$. This work aims to develop a physical model for the estimation of chlorophyll a concentration from the MODIS images in the Peruvian sea.

Keywords: chlorophyll a, satellite images, MODIS, Oceanography.

Nota de los editores. Este es un artículo que ha sido publicado en algunos sítios de internet. Hemos aceptado este artículo en vista del interés del tema y por que se requiere de una referenciación al artículo de manera permanente.

La teledetección del color del océano produce información sobre los constituyentes del agua del mar tales como la concentración de los pigmentos del fitoplancton, los sedimentos suspendidos y la sustancia amarilla [1]. Los métodos de detección y mapeado de los constituyentes del agua del mar realizado desde los aviones y los satélites han sido desarrollados exitosamente durante las últimas tres décadas. El monitoreo rutinario de la variabilidad regional y temporal de la clorofila del oceáno proporciona información sobre la producción primaria, la evaluación de los procesos de producción secundarios y sobre los órdenes mayores de producción tales como el zooplancton y la pesca marina. La clorofila-a es un indicador de la biomasa del plancton y se utiliza como un parámetro de entrada en los modelos de producción primaria y para el rastreo de corrientes, chorros y las plumas oceanográficas. La concentración de clorofila-a en una muestra de agua permite una estimación de la concentración de fitoplancton e, indirectamente, de la actividad biológica [4]. Los organismos que contienen clorofila-a se encuentra en la base de la cadena alimenticia, por lo que la determinación de las concentraciones de clorofila-a es uno de los índices claves del monitoreo de la población del fitoplancton y de la salud del sistema natural. La clorofila-a de la superficie del mar peruano puede ser medida mediante la teledetección por satélite en el rango espectral del visible. El objetivo de este trabajo es desarrollar un modelo físico para la estimación de la concentración de la clorofila-a procedente de las imágenes MODIS del mar.

*jrojasa@unmsm.edu.pe 


\section{El algoritmo de la clorofila-a caso 2}

El algoritmo de la clorofila-a caso 2 [4,7] está basado en un modelo bio-óptico semi-analítico de la reflectancia de sensoramiento remoto, $R_{r s}(\lambda)$, la cual está definida como la radiancia que sale del agua, $L_{w}(\lambda)$, dividido por la irradiancia que incide sobre la superficie del mar, $E_{d}\left(\lambda, 0^{+}\right)$. El modelo de la reflectancia de sensoramiento remoto, $R_{r s}(\lambda)$, describe un algoritmo que tiene dos variables independientes, el coeficiente de absorción debido al fitoplancton en la longitud $\lambda=675 \mathrm{~nm}, a_{\varphi}(675)$, y el coeficiente de absorción debido al material orgánico disuelto denomiando gelbstoff para $\lambda=400 \mathrm{~nm}, a_{g}(400)$. Los valores de la reflectancia $R_{r s}\left(\lambda_{i}\right)$ del sistema de procesamiento de datos MODIS se ingresan en el modelo, luego se invierte el modelo y se calculan $a_{\varphi}(675), a_{g}(400)$ y la clorofila-a. El algoritmo produce los coeficientes de absorción total, $a\left(\lambda_{i}\right), \mathrm{y}$ también permite el cálculo de los coeficientes de absorción del fitoplancton, $a_{\varphi}\left(\lambda_{i}\right)$, basado en la recuperación de $a_{\varphi}(675)$. Estos son utilizados para el cálculo de la radiación absorbida por el fitoplancton y para el cálculo de la eficiencia de la fluorescencia de la clorofila.

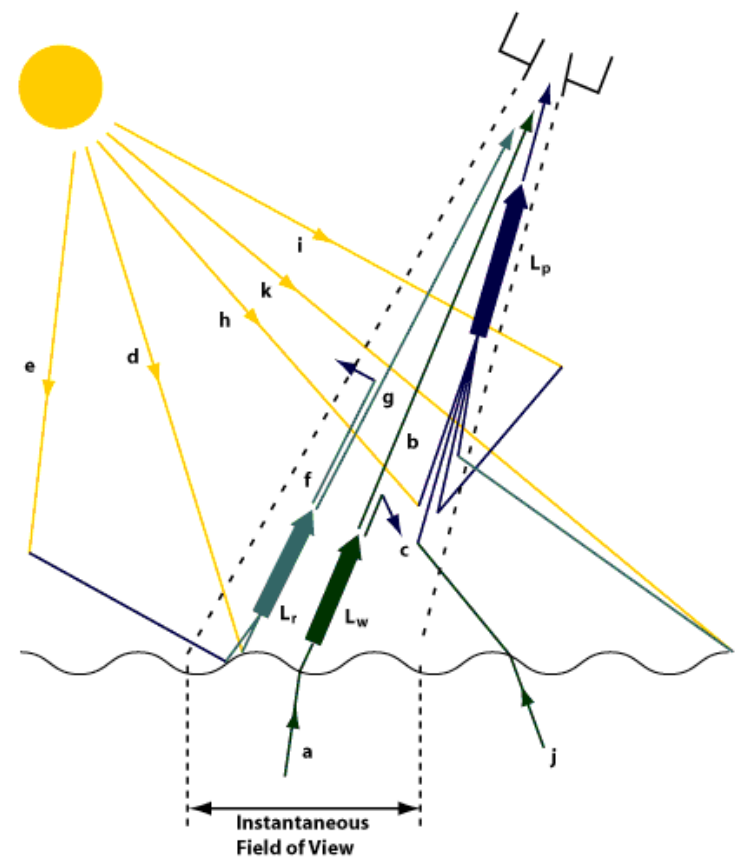

Figura 1: Modelo bio-óptico semi-analítico de la reflectancia de sensoramiento remoto [1].

El algoritmo requiere como entrada la radiancia que sale del agua, $L_{w}$, en las bandas del MODIS para las ondas oceánicas con longitud de ondas centradas en 412 , 443, 488 y 551 nm y los dominios bio-ópticos están di-

\footnotetext{
${ }^{1}$ Conocidos como las propiedades ópticas inherentes
}

señados sobre la base de la temperatura superficial del mar, que es un producto obtenido del MODIS. La reflectancia, $R_{r s}$, es obtenida de la radiancia que sale del agua, $L_{w}$, como

$$
R_{\mathrm{rs}}=\frac{L_{w}}{F_{0} t_{d} \cos \theta}
$$

donde $F_{0}$ es la irradiancia solar extraterrestre, $\theta$ es el ángulo cenit del Sol y $t_{d}$ es la transmitancia de la luz a través de la zona de interacción mar-aire. La resolución de $1000 \mathrm{~m}$ en las nuevas bandas espectrales y la cobertura diaria del MODIS permite la observación de las características oceanográficas en mesoescala para entornos costeros y estuarios que son las áreas más importantes en muchos estudios de las ciencias marinas, además de las tradicionales observaciones en el océano abierto.

En la figura 1, se ilustran los diferentes caminos de la luz en la atmósfera. El rayo $a$ es el camino de la radiancia que sale del agua, el rayo $b$ es la atenuación de la radiancia que sale del agua, el rayo $c$ es la dispersión de la radiancia que sale del agua en el campo de visión del sensor, $d$ es el rayo que incide sobre la superficie del agua, $e$ es la luz incidente sobre la superficie del agua pero fuera del campo de visión del sensor, $f$ es la luz dispersada dentro del campo de visión del sensor, $g$ es la luz dispersada fuera del campo de visión del sensor de la luz reflejada por la superficie del agua, $h$ es la luz dispersada del sol que es dirigida al sensor, $j$ es la radiancia que sale del agua originada fuera del campo de visión del sensor, pero dispersada al sensor, y finalmente, $k$ es la reflexión de la superficie fuera del campo de visión del sensor que es entonces dispersada hacia el sensor. Además, $L_{w}$ es la radiancia total que sale del agua, $L_{r}$ es la radiancia encima de la superficie del mar debido a todos los efectos de la reflexión dentro del campo de visión instantánea y $L_{p}$ es la radiancia del camino atmosférico [1,4,5.

\section{La física del problema}

Después de que la luz ingresa al océano, una parte se dispersa hacia atrás subiendo a través de la superficie. Esta luz es llamada de radiancia que sale del agua, $L_{w}(\lambda)$, y puede ser deducido desde el espacio después de eliminar los efectos atmosféricos. La magnitud, la variación espectral y la distribución angular de esta radiancia depende de los factores siguientes: los coeficientes de la absorción del agua de mar, $a(\lambda)$, de la dispersión, $b_{b}(\lambda)^{1}$, la irradiancia incidente sobre la superficie del mar, $E_{d}\left(\lambda, 0^{+}\right)$, y la distribución angular de la luz dentro del océano.

Para un mejor análisis, el agua del mar se divide en tres componentes que poseen distintas propiedades 
ópticas. Estas componentes son el agua de mar propiamente dicho en su fase líquida con sales, la fracción de partículas y la fracción disuelta. Afortunadamente, $a(\lambda)$ es simplemente igual a la suma de los coeficientes de absorción para cada componente, y $b_{b}(\lambda)$ es igual a la suma de los coeficientes de retrodispersión.

$\mathrm{Al}$ determinar cada componente, espectralmente distinto, de los coeficientes de absorción y retrodispersión, entonces se puede determinar la magnitud de cada medición de $L_{w}(\lambda)$ y $E_{d}\left(\lambda, 0^{+}\right)$, dando algunas suposiciones acerca de las distribución angular de la luz en el agua. Un punto crítico es modelar exactamente el comportamiento espectral de $a(\lambda)$ para cada componente. En cambio, el comportamiento espectral de $b_{b}(\lambda)$ no es tan dinámico.

\section{Modelo de la reflectancia de sensoramiento remoto}

El modelo de la reflectancia de sensoramiento remoto, $R_{\mathrm{rs}}$, está dado por

$$
R_{\mathrm{rs}}(\lambda)=\frac{f t^{2}}{Q(\lambda) n^{2}} \frac{b_{b}(\lambda)}{\left[a(\lambda)+b_{b}(\lambda)\right]}
$$

donde $f$ es un factor empírico cuyo valor varía entre 0.32 y $0.33, t$ es la transmitancia de la zona de interacción mar-aire, $Q(\lambda)$ es la razón entre la irradiancia y la radiancia hacia arriba, $E_{u}(\lambda) / L_{u}(\lambda)$, y $n$ es la parte real del índice de refracción del agua de mar. Al hacer tres aproximaciones, la Ec. (2) puede ser simplificada enormemente. Veamos, la primera es asumir que $f / Q(\lambda)$ es independiente de $\lambda$ y $\theta_{0}$ para todas las bandas MODIS de interés; la segunda es que el término $t^{2} / n^{2}$ es, aproximadamente, igual a 0.54 ; y la tercera, $b_{b}(\lambda)$ es mucho mas pequeña que $a(\lambda)$ y puede ser eliminado del denominador de la Ec. (2). Estas tres aproximaciones conducen a

$$
R_{\mathrm{rs}}(\lambda) \approx \text { constante } \frac{b_{b}(\lambda)}{a(\lambda)}
$$

donde el término constante no cambia con respecto a $\lambda$ y $\theta_{0}$. El valor de la constante no es relevante para el algoritmo ya que este usa razones espectrales de $R_{\mathrm{rs}}(\lambda)$ y el término constante se factoriza. Los términos $b_{b}(\lambda) \mathrm{y}$ $a(\lambda)$ serán divididos en varios términos separados. Cada término será descrito empíricamente. Las ecuaciones son escritas en una forma general, donde los parámetros obtenidos empíricamente y que describen cada término, son escritos como variables y los valores actuales de los parámetros que son usados en el algoritmo son mostrados en las tablas 1 y 2 .

\begin{tabular}{ccccc}
\hline$\lambda$ & 412 & 443 & 488 & 551 \\
\hline$b_{b}\left(10^{-3} \mathrm{~m}^{-1}\right)$ & 3.341 & 2.406 & 1.563 & 0.929 \\
$a_{w}\left(10^{-3} \mathrm{~m}^{-1}\right)$ & 4.8 & 7.42 & 16.32 & 59.1 \\
$a_{0}$ & 2.2 & 3.59 & 2.27 & 0.42 \\
$a_{1}$ & 0.75 & 0.8 & 0.59 & -0.22 \\
$a_{2}$ & -0.5 & -0.5 & -0.5 & -0.5 \\
$a_{3}\left(10^{-2}\right)$ & 1.12 & 1.12 & 1.12 & 1.12 \\
\hline
\end{tabular}

Tabla 1: Parámetros que dependen de la longitud de onda para el algoritmo clorofila semianalítico para regiones sin pigmentos empaquetados.

\begin{tabular}{rr|rr|cr}
\hline$X_{0}$ & -0.00182 & $S$ & 0.0225 & $c_{0}$ & 0.2818 \\
$X_{1}$ & 2.058 & $p_{0}$ & 51.9 & $c_{1}$ & -2.783 \\
$Y_{0}$ & -1.13 & $p_{1}$ & 1 & $c_{2}$ & 1.863 \\
$Y_{1}$ & 2.57 & & & $c_{3}$ & -2.387 \\
\hline
\end{tabular}

Tabla 2: Parámetros independientes de la longitud de onda para el algoritmo clorofila semianalítico para regiones sin pigmentos empaquetados.

\section{El término de retrodispersión}

El coeficiente de retrodispersión total, $b_{b}(\lambda)$, puede ser expresado como

$$
b_{b}(\lambda)=b_{b w}(\lambda)+b_{b p}(\lambda)
$$

donde los subíndices $b w$ y $b p$ se refieren al agua y a las partículas, respectivamente. El coeficiente $b_{b w}(\lambda)$, es constante y conocido. El coeficiente $b_{b p}(\lambda)$, es modelado como

$$
b_{b p}(\lambda)=X\left[\frac{551}{\lambda}\right]^{Y}
$$

La magnitud de la retrodispersión de las partículas es indicado por $X=b_{b p}(551)$, mientras que $Y$ describe la forma espectral de la retrodispersión de las partículas. Empíricamente se determinó los valores de $X$ e $Y$ por un modelo de inversión usando una fórmula similar a la Ec. (5). Los valores de $X$ e $Y$ fueron comparados con los valores de $R_{\mathrm{rs}}(\lambda)$ medidos en cada estación con el propósito de determinar las relaciones empíricas de $X$ e $Y$ como una función de $R_{\mathrm{rs}}(\lambda)$ en una o más longitudes de onda del MODIS. Estas relaciones empíricas son describen con mayor detalle más adelante.

\section{La expresión para $X$}

la retodipersión de las partículas se determina mediante

$$
X=X_{0}+X_{1} R_{r s}(551)
$$

donde $X_{0}=-0.00182$ y $X_{1}=2.058$, para $n=53$ y $r^{2}=0.96$, son constantes obtenidas empíricamente. Si $X$ sale negativo de la ecuación (6), deberá fijarse como cero. 


\section{La expresión para $Y$}

La forma espectral de la retrodispersión de las partículas $Y$ fue hallado variando la razón $R_{r s}(443) / R_{r s}(488)$. Las variaciones en los valores del numerador y el denominador de esta razón son determinadas principalmente por la absorción del fitoplancton y el colorido material orgánico disuelto, Coloric Disolved Organic Material, CDOM. La absorción atribuible al agua es el mismo y bajo en ambas longitudes de onda. Así, la absorción del fitoplancton y el CDOM covarien, la razón espectral de los coeficientes de absorción $a(443) / a(488)$, será solo débilmente dependiente de la concentración del pigmento, y la razón espectral de los coeficientes de retrodispersión deberán tener un efecto significativo sobre la razón espectral de la $R_{r s}$. Por lo tanto $Y$ se representa como

$$
Y=Y_{0}+Y_{1} \frac{R_{r s}(443)}{R_{r s}(448)}
$$

Una función lineal de $R_{r s}(443) / R_{r s}(448)$ donde $Y_{0}$ y $Y_{1}$ son constantes obtenidas empíricamente y además $Y_{0}=-1.13$ y $Y_{1}=2.57$ para $n=22, r^{2}=0.59$. Cuando $Y$ es negativo, la ecuación (7) se establece a cero.

\section{El término de absorción}

El coeficiente de absorción total puede ser expandido como

$$
a(\lambda)=a_{w}(\lambda)+a_{\varphi}(\lambda)+a_{d}(\lambda)+a_{g}(\lambda),
$$

donde los subíndices $w, \varphi, d, \mathrm{y} g$ se refieren al agua, al fitoplancton, al detritus y al CDOM, observando que $g$ significa gelbstoff. Los valores de $a_{w}(\lambda)$ son conocidos y las expresiones para $a_{\varphi}(\lambda), a_{d}(\lambda)$ y $a_{g}(\lambda)$ lo desarrollamos a continuación.

\section{Determinación de $a_{\varphi}$}

La forma del espectro de $a_{\varphi}(\lambda)$ para una masa de agua en particular cambiará debido al efecto de empaquetamiento del pigmento, es decir, el aplanamiento de los picos de la absorción es debido al autosombreado con el incremento de las concentraciones del pigmento intracelular y el gran tamaño de celdas y los cambios en la composición del pigmento. Para un dominio en particular, las curvas de $a_{\varphi}(\lambda)$ son medidas normalizadas a $a_{\varphi}(675)$ que reduce el rango dinámico y resulta en una variación suave para $a_{\varphi}(\lambda) / a_{\varphi}(675)$ versus $a_{\varphi}(675)$ en las bandas MODIS centradas en datos con $\lambda=412,443,488, y 551 \mathrm{~nm}$ para los dos regímenes subtropical de luz intensa. Como la clorofila-a es proporcional a $a_{\varphi}(675)$ se muestra cómo la absorción específica de la clorofila cambia con la concentración de la clorofila. Una función tangente hiperbólica fue escogida para modelar esta relación con el fin de asegurar que el valor de $a_{\varphi}(\lambda) / a_{\varphi}(675)$ se aproxime a una asíntota en los valores más altos o valores más bajos de $a_{\varphi}(675)$. Usando las escalas logarítmicas para ambas variables expresamos la siguiente ecuación del modelo para $a_{\varphi}(\lambda)$ como una función de $a_{\varphi}(675)$

$$
\begin{aligned}
a_{\varphi}(\lambda) & =a_{0}(\lambda) a_{\varphi}(675) \times \\
& \exp \left[a_{1}(\lambda) \tanh \left(a_{2}(\lambda) \ln \left[\frac{a_{\varphi}(675)}{a_{3}(\lambda)}\right]\right)\right],
\end{aligned}
$$

donde los parámetros $a_{0}(\lambda)$ hasta $a_{3}(\lambda)$ son determinados empíricamente para cada longitud de onda de interés del MODIS. El parámetro $a_{0}(\lambda)$ es el más importante por cuanto es directamente proporcional a $a_{\varphi}(\lambda)$. Por simplicidad, solo $a_{0}(\lambda)$ y $a_{1}(\lambda)$ son variados para parametrizar $a_{\varphi}(\lambda)$, con $a_{2}(\lambda)=-0.5$ y $a_{3}(\lambda)=0.0112$ como valores constantes.

\section{Determinación de $a_{d}$ y $a_{g}$}

Los coeficientes $a_{d}(\lambda)$ y $a_{g}(\lambda)$ pueden ser ajustados de la forma $a_{x}(\lambda)=a_{x}(400) \exp \left[-S_{x}(\lambda-400)\right]$ donde los subíndices $x$ se refiere a $d$ o $g$. Debido a esta similaridad en la forma espectral, esos términos no pueden ser espectralmente separados por los canales MODIS, así el término $a_{d}(\lambda)$ es combinado operacionalmente con $a_{g}(\lambda)$, y las absorciones detritales y los CDOM son representados por $a_{g}(\lambda)$ de la forma

$$
a_{g}(\lambda)=a_{g}(400) \exp [-S(\lambda-400)],
$$

donde $S$ es determinado empíricamente. Muchos investigadores han reportado que $S_{d}=0.011$, en promedio. Para los cruceros GOMEX y COLOR, un valor promedio de $S_{g}=0.017 \mathrm{~nm}^{-1}$ fue medido. Como nota final sobre el modelo $R_{r s}$, las ecuaciones (5-9) son escritos en una forma general para enfatizar que los valores de los parámetros $X_{0}, X_{1}, Y_{0}, Y_{1}, a_{0}, a_{1}$ no son absolutos. Ellos deben ser actualizados y cambiados cuando más datos están disponibles. Estos parámetros pueden también ser cambiados por la región y la estación para optimizar el rendimiento del algoritmo.

\section{Invirtiendo el modelo}

Todas las piezas del modelo de reflectancia están ahora en su lugar. Desde las ecuaciones (2-4) y (5$9), R_{r s}(\lambda)$ puede ser expresado solamente como una función del término constant2, $R_{r s}(443), R_{r s}(448)$, $R_{r s}(551), a_{\varphi}(675)$, y $a_{g}(675)$, dando los valores para los parámetros para $X_{0}, X_{1}, Y_{0}, Y_{1}, a_{0}(\lambda), a_{1}(\lambda)$, y el parámetro $S, L_{w n}(\lambda)$ del MODIS puede ser convertido en $R_{r s}(\lambda)$ como fue mencionado anteriormente. Entonces, para cada píxel, la ecuación del modelo $R_{r s}$ puede 
ser escrita para cada uno de las 5 bandas MODIS disponibles produciendo cinco ecuaciones con tres incógnitas: el término constante, $a_{\varphi}(675)$, y $a_{g}(400)$. Usando las razones espectrales de $R_{r s}(\lambda)$ elimina el término constante, ya que es independiente de la longitud de onda. En principio, dos ecuaciones de razón espectral pueden ser usadas para resolver las dos incógnitas restantes $a_{\varphi}(675)$, y $a_{g}(400)$. Basados en la forma de la curva de absorción para el fitoplancton versus detritus y CDOM, las ecuaciones que usan razones espectrales de 412:443 y 443:551 para $R_{r s}(\lambda)$ deberá proporcionar una buena separación de las dos contribuciones de absorción. Nuestras dos ecuaciones resultantes son

$$
\left.\begin{array}{l}
\frac{R_{r s}(412)}{R_{r s}(443)}=\frac{b_{b}(412)}{b_{b}(443)} \frac{a(443)}{a(412)} \\
\frac{R_{r s}(443)}{R_{r s}(551)}=\frac{b_{b}(443)}{b_{b}(551)} \frac{a(551)}{a(443)}
\end{array}\right\}
$$

El miembro derecho de cada ecuación es una función de $a_{\varphi}(675), a_{g}(400), R_{r s}(443), R_{r s}(488)$ y $R_{r s}(551)$. Los valores de $R_{r s}$ son proporcionados como entrada del modelo, ahora tenemos dos ecuaciones con dos incógnitas. Las ecuaciones son resueltas algebraicamente para proporcionar los valores de $a_{\varphi}(675)$ y $a_{g}(400)$. Estas ecuaciones se resuelven mediante un método computacional. Para aguas con altas concentraciones de CDOM y de clorofila, los valores de $L_{w}(412)$ y $L_{w}(443)$ son pequeños, y el algoritmo semi-analítico no puede funcionar apropiadamente [3, 6, 8, 12, El algoritmo bioóptico OC3M para MODIS está dado por

$$
\begin{gathered}
R_{L}=\log _{10}\left(\operatorname{máx}\left[\frac{R_{r s}(443)}{R_{r s}(551)}, \frac{R_{r s}(488)}{R_{r s}(551)}\right]\right) \\
\log _{10} C_{a}=0.283-2.753 \times R_{L}+0.659 \times R_{L}^{2}+ \\
0.649 \times R_{L}^{3}-1.403 \times R_{L}^{4} .
\end{gathered}
$$

El algoritmo empírico para MODIS OC3M que se encuentra operativo es

$$
C_{a}=10.0^{0.2830-2.753 R_{3 M}+1.457 R_{3 M}^{2}+0.659 R_{3 M}^{3}-1.403 R_{3 M}^{4}}
$$

donde

$$
R_{3 M}=\log _{10}\left(R_{550}^{443}>R_{550}^{490}\right)
$$

El algoritmo depende del máximo empírico de la razón de las tres bandas MODIS. 7, 13,

\section{Conclusiones}

En el presente trabajo hemos desarrollado un modelo físico que cuantifica la reflectancia producida por la clorofila-a que son captadas en las imágenes MODIS. Nuestro modelo consigue determinar la concentración de clorofila-a y CDM que se encuentran en la superficie del mar.

\section{Referencias}

[1] I. S. Robinson, Satellite observations of ocean colour, Phil. Trans. Royal Soc. of London A, Volume 309, 338 (1983).

www.physics.miami.edu/ chris/envr_optics.html

[2] H. R. Gordon y A. Morel; Remote Assessment of Ocean Color for Interpretation of Satellite Visible Imagery: A Review, Springer-Verlag, New York (1983).

[3] H. R. Gordon, O. B. Brown, R. H. Evans, J. W. Brown, R. C. Smith, K. S. Baker y D. K. Clark; A Semi-analytic Radiance Model of Ocean Color, J. Geophys. Res. 93, D9, 10909 (1988).

[4] K. L. Carder, F. R. Chen, Z. Lee, S. K. Hawes y J. P. Cannizzaro; Case 2 Chlorophyll a, MODIS Ocean Science Team, Algorithm Theoretical Basis Document ATBD 19 (2003).

[5] K. L. Carder, F. R. Chen, Z. P. Lee y S. K. Hawes; Semianalytic Moderate-Resolution Imaging
Spectrometer algorithms for chlorophyll $a$ and $a b$ sorption with bio-optical domains based on nitratedepletion temperatures, J. Geophys. Res. 104, C3, 5403 (1999).

[6] H. R. Gordon, D. K. Clark, J. W. Brown, O. B. Brown, R. H. Evans y W. W. Broenkow; Phytoplankton pigment concentrations in the Middle Atlantic Bight: Comparison of ship determinations and CZCS estimates, Appl. Opt. 22, 20 (1983).

[7] K. L. Carder, S. K. Hawes, Z. Lee y F. R. Chen; MODIS: Case 2 chlorophyll a algorithm, ATBD-19, (1997).

[8] H. R. Gordon y M. Wang; Retrieval of waterleaving radiance and aerosol optical thickness over the ocean with SeaWiFS: A preliminary algorithm, Appl. Opt., 33, 443 (1994).

[9] H. R. Gordon, O. B. Brown y M. M. Jacobs; Computed relationships between the inherent and apparent optical properties of a flat homogeneous ocean, Appl. Opt., 14, 417 (1975). 
[10] H. R. Gordon, MODIS Normalized Water-leaving Radiance Algorithm Theoretical Basis Document, August 15 (1996). eospso.gsfc.nasa.gov/atbd/modis/atbdmodl7new.html

[11] J. H. Jerome, R. P. Bukata y J. E. Burton; Utilizing the components of vector irradiance to estimate the scalar irradiance in natural waters, Appl. Opt., 27, 4012 (1988).
[12] J. E. O'Reilly, S. Maritorena, B. G. Mitchell, D. A. Siegel, K. L. Carder, S. A. Garver, M. Kahru y C. McClain; Ocean color chlorophyll algorithms for SeaWiFS, J. Geophys. Res. 103, C11, 24937 (1998).

[13] S. Martin, An Introduction Ocean Remote Sensing, Cambridge University Press, Cambridge (2002). 